\title{
Diffusional kinetics of SiGe dimers on Si(100) using atom-tracking scanning tunneling microscopy
}

\author{
X.R. Qin ${ }^{1}$, B. S. Swartzentruber ${ }^{2}$, M. G. Lagally ${ }^{1}$ \\ ${ }^{1}$ University of Wisconsin-Madison, Madison WI 53706 \\ ${ }^{2}$ Sandia National Laboratories, Albuquerque, NM 87185-1421
}

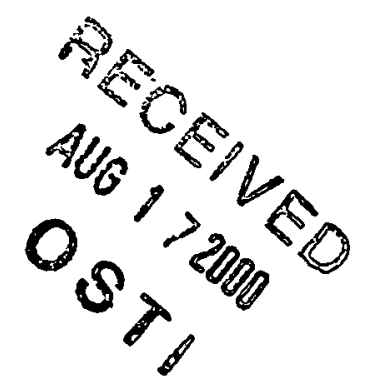

\begin{abstract}
Quantitative measurements of the diffusion of adsorbed mixed Ge-Si dimers on the $\mathrm{Si}(100)$ surface have been made as a function of temperature using atom-tracking scanning tunneling microscopy. These mixed dimers are distinguishable from pure Si-Si dimers by their characteristic kinetics - a 180-degree rotation between two highly buckled configurations. At temperatures at which the mixed dimers diffuse, atomic-exchange events occur, in which the Ge atom in the adsorbed dimer exchanges with a substrate $\mathrm{Si}$ atom. Re-exchange can also occur when the diffusing Si-Si dimer revisits the original site of exchange.
\end{abstract}

PACS numbers: 68.10.Jy, 61.16.Ch, 68.35.Fx, 68.35.Bs 


\section{DISCLAIMER}

This report was prepared as an account of work sponsored by an agency of the United States Government. Neither the United States Government nor any agency thereof, nor any of their employees, make any warranty, express or implied, or assumes any legal liability or responsibility for the accuracy, completeness, or usefulness of any information, apparatus, product, or process disclosed, or represents that its use would not infringe privately owned rights. Reference herein to any specific commercial product, process, or service by trade name, trademark, manufacturer, or otherwise does not necessarily constitute or imply its endorsement, recommendation, or favoring by the United States Government or any agency thereof. The views and opinions of authors expressed herein do not necessarily state or reflect those of the United States Government or any agency thereof. 


\section{DISCLAIMER}

Portions of this document may be illegible in electronic image products. Images are produced from the best available original document. 
As the dimensions of artificially structured materials decrease, the role of the interfaces becomes increasingly important in determining physical and electronic properties. In particular, interface roughness between two dissimilar materials can affect a host of properties, among them the strength of the material, electron and photon scattering at the interfaces of electronic and optoelectronic materials, and the magnetic properties of magnetic multilayers. One component of interface roughness in layered thin-film systems is the formation of ill-defined interfaces by atomic intermixing during growth. The details of the intermixing are determined by the interplay of the thermodynamic properties of the interfaces and the transport and reaction kinetics of individual atoms, dimers, and small clusters.

In semiconductor systems, $\mathrm{Ge}$ or $\mathrm{SiGe}$ growth on $\mathrm{Si}(100)$ serves as a model heteroepitaxial system and has attracted considerable attention because of the use and potential use of GeSi films in fast electronic devices, and because of the compatibility of this system with Si processing. In order to follow the kinetics of individual atoms or small clusters in a multicomponent system, a technique with both atomic spatial resolution and atomic chemical sensitivity is needed. Scanning tunneling microscopy (STM) provides atomic-resolution images of surface structures:jalthough it is, in general, not chemically sensitive. It is particularly difficult to differentiate $\mathrm{Ge}$ and $\mathrm{Si}$ because of their similar electronic structures (l). Nevertheless, much has already been learned about the atomic-scale surface morphology, surface stress, and surface intermixing during the initial stages of growth of $\mathrm{Ge}$ on $\mathrm{Si}(100)$ (2). Lacking is direct information on how kinetic processes such as diffusion and intermixing occur.

In this Letter, we demonstrate, using atom-tacking STM (AT-STM), unprecedented spatial and time resolution in the study of dynamic processes of atomic intermixing of $\mathrm{Ge}$ on $\mathrm{Si}(100)$. The large increase in time and spatial resolution of AT-STM relative to conventional 
STM imaging $(3,4)$, allows us to identify the difference between adsorbed mixed Ge-Si dimers and pure Si-Si dimers based on their distinct kinetic behavior. Along with determining the activation barriers for the Ge-Si dimers to rotate and diffuse, we also directly observe intermixing events in which the Ge atom in the adsorbed dimer exchanges with a Si atom in the substrate.

The experiments were performed in an ultrahigh-vacuum variable-temperature AT-STM system $(3,4)$ with a base pressure below $8 \times 10^{-11}$ torr. The Si substrates were cleaned by resistive heating in the conventional manner by degassing at $700^{\circ} \mathrm{C}$, flashing to $1200^{\circ} \mathrm{C}$ for $\sim 1$ minute, and then radiation cooling (5). Ge was deposited onto the substrate from a resistively heated tungsten wire basket by opening an intervening shutter for 3-5 seconds, resulting in the deposition of about $1 \%$ of a monolayer of Ge atoms. All of the atom-tracking data presented in this work were obtained using a tunneling sample bias of $-2.5 \mathrm{~V}$ at $\sim 0.04 \mathrm{nA}$ (๑).

The silicon (100) surface consists of rows of dimers that form a $2 \times 1$ reconstruction relative to the $1 \mathrm{x} 1$ bulk lattice structure in order to reduce the surface free energy. After $\mathrm{Ge}$ deposition we observe small islands and clusters of atoms on the surface as well as isolated adsorbed dimers that sit on top of the substrate dimer rows. Among these isolated dimers, we observe many in which one of the atoms of the dimer is much higher than the other, i.e., highly buckled, with their dimer bond parallel to the substrate dimer row direction. At room temperature these dimers appear to "rock" between two configuration states with 180-degree rotational symmetry at a single lattice site with an average time between events of $\sim 3 \mathrm{~s}$. First one side of the dimer appears higher and then the other. The rates of rocking back and forth are exactly the same. We show STM images of a rocking dimer in its two configuration states in Fig. 1. This room temperature behavior is clearly distinct from the behavior of pure Si-Si dimers, which 
rotate between two orthogonal orientations with complete mirror symmetry $(4,7,8)$. We conclude that the rocking dimers must therefore contain Ge.

We will show that these rocking dimers are comprised of a Ge-Si pair rather than pure Ge-Ge. That conclusion is certainly consistent with theory. Recent $a b$ initio theoretical calculations of pure Ge-Ge dimer diffusion on $\mathrm{Si}(100)$ yield a diffusion barrier of $0.77 \mathrm{eV}(9)$, which would lead to a diffusion rate several orders of magnitude faster than what we measure. The measured diffusion rate for a rocking dimer is, in fact, very similar to that for a pure Si-Si dimer. In addition, Ko et al. calculate an extremely-low-activation-barrier path for the formation of adsorbed mixed Ge-Si dimers $(0.1 \mathrm{eV})$ from the deposition of $\mathrm{Ge}(10)$. They find that when two diffusing Ge atoms meet, an exchange process takes place in which one of the Ge atoms exchanges with a substrate $\mathrm{Si}$ atom, leading to a mixed dimer in the substrate surface and an adsorbed mixed dimer (10). Experimental evidence for the mixed composition of the dimer is presented below.

Because the rocking images have 180-degree rotational symmetry and the kinetics of rocking back and forth are the same, the dynamic behavior of this mixed dimer is actually due to the rotation of the dimer about the surface normal and not simply a shift in which atom is in the higher-tilt position, which would lead to asymmetric behavior. Calculations of the adsorbed mixed Ge-Si dimer on $\mathrm{Si}(100)$ find a strongly-buckled structure with the Ge atom in the up position (11).

In Figs. $2 \mathrm{~A}$ and $2 \mathrm{~B}$ we display two STM images at a substrate temperature of $70^{\circ} \mathrm{C}$ taken 35 seconds apart that clearly show the diffusion of an adsorbed dimer. At this temperature the dimer hops about once every 5 seconds. To measure the precise details of its kinetic behavior we employ the atom-tracking technique $(3,4)$, which increases the time resolution of our instrument 
by several orders of magnitude over conventional STM imaging. During atom tracking the STM probe tip is locked on to the highest point of the diffusing dimer using lateral $\mathrm{XY}$ feedback. The $\mathrm{XY}$ position of the tip is then monitored as a function of time. Fig. $2 \mathrm{C}$ shows the $\mathrm{XY}$ scatter plot of 30,000 data points acquired over 185 seconds. In Fig. 2D we plot the $\mathrm{X}$ coordinate as a function of time. We see that the dimer visited six lattice sites during the data acquisition. The lattice sites are separated by a distance $a_{0}=3.84 \AA$. At each lattice site the mixed Ge-Si dimer rotates between its two stable configurations (i.e., with the Ge atom on the left or right side) on a time scale much faster than the hopping rate. When the dimer rotates, the lateral feedback adjusts the tip position to be over the highest point, which is the location of the Ge atom. This rotation thus manifests itself as two groups of a high density of scatter points separated by a distance, $r$, at each lattice site (12). At $70^{\circ} \mathrm{C}$ the rotation events occur on a time scale of $\sim 40 \mathrm{~ms}$, which is close to the tracking limit of our instrument $(\sim 5 \mathrm{~ms})$, resulting in data points located between the two stable positions. The highest temperature at which we can resolve the individual rotation events is therefore $\sim 70^{\circ} \mathrm{C}$.

To extract the activation barriers for hopping and rotation, we measure their rates as a function of temperature. The event rate is equal to the inverse of the measured average residence time, $\tau$, i.e., the time between events. The event rates follow the Arrhenius relation, $1 / \tau=\nu_{0}$ $\exp \left(-E_{a} / k T\right)$, in which $E_{a}$ is the activation energy and the prefactor, $v_{\mathrm{o}}$, is the attempt frequency. In Fig. 3 we show the Arrhenius plots for the hopping and rotation kinetics. The measured hopping and rotation barriers are respectively $1.01 \pm 0.09 \mathrm{eV}$ and $0.82 \pm 0.01 \mathrm{eV}$, with frequency prefactors of $10^{14.0 \pm 1.5} \mathrm{~Hz}$ and $10^{12.9 \pm 0.2} \mathrm{~Hz}$. The measured hopping barrier for these mixed Ge-Si dimers is very similar to that measured previously for pure Si-Si dimers $(3,13)$. Recent first-principles calculations of the rotation of mixed Ge-Si dimers on the Si(100) surface 
obtain a barrier of $0.7 \mathrm{eV}(14)$, close to our measured value.

In these measurements of the rotation of the mixed Ge-Si dimers, we are not able to detect the existence of a metastable configuration state in which the adsorbed dimer bond direction is perpendicular to the substrate dimer row direction. As shown in previous work, this 90-degree rotated metastable configuration does exist for adsorbed Si-Si dimers with a configuration energy $0.06 \mathrm{eV}$ higher than the most stable orientation (4). That we cannot detect this state for the mixed Ge-Si dimer, even at room temperature, puts a lower limit on its configuration energy. With a configuration energy only $0.15 \mathrm{eV}$ higher than the stable state, the average residence time for the dimer in the 90 -degree orientation would be about $40 \mathrm{~ms}$ and the average residence time in a metastable state $0.20 \mathrm{eV}$ higher in energy would be $6 \mathrm{~ms}$.

Because of the distinct behavior of the Ge-Si dimer, it is easy to determine the nature of an adsorbed dimer from its tracking data. The track position is locally smeared for a mixed dimer diffusing along a substrate row because of the dimer's 180-degree rotation. The behavior of a mixed dimer can change abruptly. In Fig. 4A we show the tracking data for the same dimer plotted in Fig. 2. Near the end of the plot, the smeared band becomes suddenly very narrow (arrow) as the 180-degree rotation is no longer evident. This is clear in the expanded plot directly below. This change in behavior signals a change in the composition of the adsorbed dimer. In an STM image taken immediately after this change (Fig. 4B), we see that the substrate dimer row beneath the adsorbed dimer is buckled, whereas originally it was not buckled (Figs. 2A and 2B). Buckling of the Si surface dimer rows is the typical signature of $\mathrm{Ge} / \mathrm{Si}$ intermixing $(I)$. The subsequent behavior of the resulting adsorbed dimer is the same as that of a pure Si-Si dimer $(3,4)$. Atom-tracking measurements of the hop rate of the dimer, assuming a prefactor of $10^{13} \mathrm{~Hz}$, yield a hopping barrier of $0.96 \mathrm{eV}$, very similar to that of pure-Si-dimer diffusion $(3,13)$. The 
abrupt change in the behavior of the diffusing dimer signals an intermixing event in which the Ge atom in the original adsorbed Ge-Si dimer exchanges with a substrate $\mathrm{Si}$ atom to form a mixed substrate dimer and an adsorbed pure Si-Si dimer.

This exchange process is completely reversible. Fig. $4 \mathrm{C}$ shows tracking data from a different dimer acquired at $100^{\circ} \mathrm{C}$. At the beginning of the data set the dimer displays the distinct behavior of a mixed Ge-Si dimer. At time, $t_{1}$, an exchange event occurs and the adsorbed dimer displays the character of a pure $\mathrm{Si}-\mathrm{Si}$ dimer. It diffuses until time, $\mathrm{t}_{2}$, at which time a re-exchange event occurs at exactly the same lattice site as the original event. The same Ge atom initially exchanged with a substrate $\mathrm{Si}$ is re-exchanged into the diffusing dimer, which again displays the behavior of a mixed Ge-Si dimer. Ko et al. (10) calculate only a small configuration energy difference between an adsorbed mixed Ge-Si dimer on a pure substrate and an adsorbed pure SiSi dimer with a mixed dimer in the substrate. Although we do not have enough statistics to extract a meaningful barrier for the Ge atom in an adsorbed $\mathrm{Ge}-\mathrm{Si}$ dimer to exchange with a substrate atom, it is clear that the exchange barrier must be larger than the measured diffusion barrier, because the dimer hops many times before an exchange event occurs.

We point out two features evident in the tracking data during an exchange event. First, the intermixing events always occur in concert with hopping events. We have never observed an event in which the rotation character of the adsorbed Ge-Si dimer switches to that of a pure $\mathrm{Si}-\mathrm{Si}$ dimer and the dimer remains at a single lattice site. Because the time scale of the actual event is many orders of magnitude faster than the resolution of our instrument, we cannot determine whether it is the exchange event that triggers the diffusion event or vice versa.

Second, the newly formed adsorbed $\mathrm{Si}$-Si dimer at the site right next to the intermixing event is tilted, with the atom farthest from the (now substrate) Ge in the up position. This can be 
seen by careful inspection of the tracking positions in Fig. 4A as well as comparison of the tracking positions labeled $\mathrm{x}_{1}, \mathrm{x}_{2}$, and $\mathrm{x}_{3}$ in Fig. $4 \mathrm{C}$ with the smeared locations of the Ge-Si dimer after re-exchange at the right side of the figure. The buckling asymmetry is greatest at the lattice site right next to the exchange site and falls off very rapidly at farther sites. These two observations provide valuable points of comparison for further theoretical studies of the detailed intermixing kinetics and energetics.

By applying atom-tracking STM to the study of Ge-Si dimer diffusion, we are able to measure real-time atomic intermixing, determining directly the time and the lattice site at which the intermixing occurs. We are able to do so because the atomic-scale behavior of the adsorbed dimers is distinctly related to their chemical composition, and therefore can be used to identify them. Using this novel approach of determining chemical composition by monitoring subtle adsorbate behavioral characteristics will be very useful in the atomic and molecular-level study of more complicated chemical systems in which chemical reactions occur.

We acknowledge useful discussions with Feng Liu (UW-Madison) and Roland Stumpf ( Sandia National Laboratories (SNL)). SNL is a multiprogram laboratory operated by Sandia Corporation, a Lockheed Martin Company, for the US DOE under Contract DE-AC0494AL85000. This work was supported by SNL (Grant No. AS-1 168), and by NSF (Grants No. DMR93-04912 and No. DMR9632527). 


\section{References:}

1. X. R. Qin, B. S. Swartzentruber, and M. G. Lagally, Phys. Rev. Lett. 84, 4645 (2000).

2. For a review, see F. Liu, F. Wu, and M. G. Lagally, Chem. Rev. 97, 1045 (1997)

3. B. S. Swartzentruber, Phys. Rev. Lett. 76, 459 (1996).

4. B. S. Swartzentruber, A. P. Smith, and H. Jonsson, Phys. Rev. Lett. 77, 2518 (1996).

5. B. S. Swartzentruber, Y. W. Mo, M. B. Webb, and M. G. Lagally, J. Vac. Sci. Technol. A7, 2901 (1989).

6. The electric field induced by the tip is not a dominant factor in the dimer behavior. For sample biases in the range from $-3.0 \mathrm{~V}$ to $-1.8 \mathrm{~V}$, the activation barrier changes by $<10$ $\mathrm{meV}$. We thus use sample bias of $-2.5 \mathrm{~V}$ for our experiments.

7. Z. Zhang, F. Wu, H. J. W. Zandvliet, B. Poelsema, H. Metiu, and M. G. Lagally, Phys. Rev. Lett. 74, 3644 (1995).

8. P. J. Bedrossian, Phys. Rev. Lett. 74, 3648 (1995).

9. Z. Y. Lu, C. Z. Wang, and K. M. Ho (private communication)

10. Y. J. Ko, K. H. Park, J. S. Ha, and W. S. Yun, Phys. Rev. B 60, 8158(1999).

11. R. R. Stumpf (private communication)

12. Although the exact value of $r$ may change for different tips, it is always less than the lattice constant.

13. B. Borovsky, M. Krueger, and E. Ganz, Phys. Rev. B 59, 1598(1999).

14. Z. Y. Lu, C. Z. Wang, Feng Liu, and K. M. Ho (to be published) 


\section{Figure Captions:}

Fig. 1 Empty-state STM images of an adsorbed dimer sitting on top of a Si(100) substrate dimer row after Ge deposition. The adsorbed dimer appears to "rock" between two tilted configurations (A and B) at room temperature. (C) The dimer consists of two atoms (shaded) with their bond parallel to the substrate dimer row. Larger size means tilted upward.

Fig. 2 Motion of an adsorbed dimer. (A and B) Filled-state STM images of an adsorbed dimer (arrows) diffusing at $70^{\circ} \mathrm{C}$ along a substrate dimer row that is unbuckled and without defects. (C) $\mathrm{X}-\mathrm{Y}$ scatter plot of the atom-track data, showing the dimer lattice site visitations (with lattice spacing $\mathrm{a}_{\mathrm{o}}=3.84 \AA$ ) and the two configuration states (separated by $\mathrm{r}$ ) at each lattice site. (D) $\mathrm{X}$ time-line plot shows the dimer position as a function of time.

Fig. 3 Arrhenius plots of the rotation and hopping rates. The error bars reflect the statistics related to the number of events at each temperature. The activation barriers are $1.01 \pm 0.09 \mathrm{eV}$ and $0.82 \pm 0.01 \mathrm{eV}$ for hopping and rotation, respectively.

Fig. 4 Demonstration that atomic exchange occurs during dimer diffusion. (A) Time-line plot $\left(70^{\circ} \mathrm{C}\right)$ of the dimer position showing the exact time of an atom exchange event (arrow), when the characteristic behavior of the Ge-Si dimer abruptly changes to that of a Si-Si dimer. (B) Filled-state STM image after the atom exchange event that puts Ge into the substrate, showing the buckling of the substrate dimer rows near the event location. (C) Time-line plot of a different dimer $\left(100^{\circ} \mathrm{C}\right)$ showing an initial atom exchange event at $t_{1}$ and a subsequent re-exchange event at $t_{2}$. 

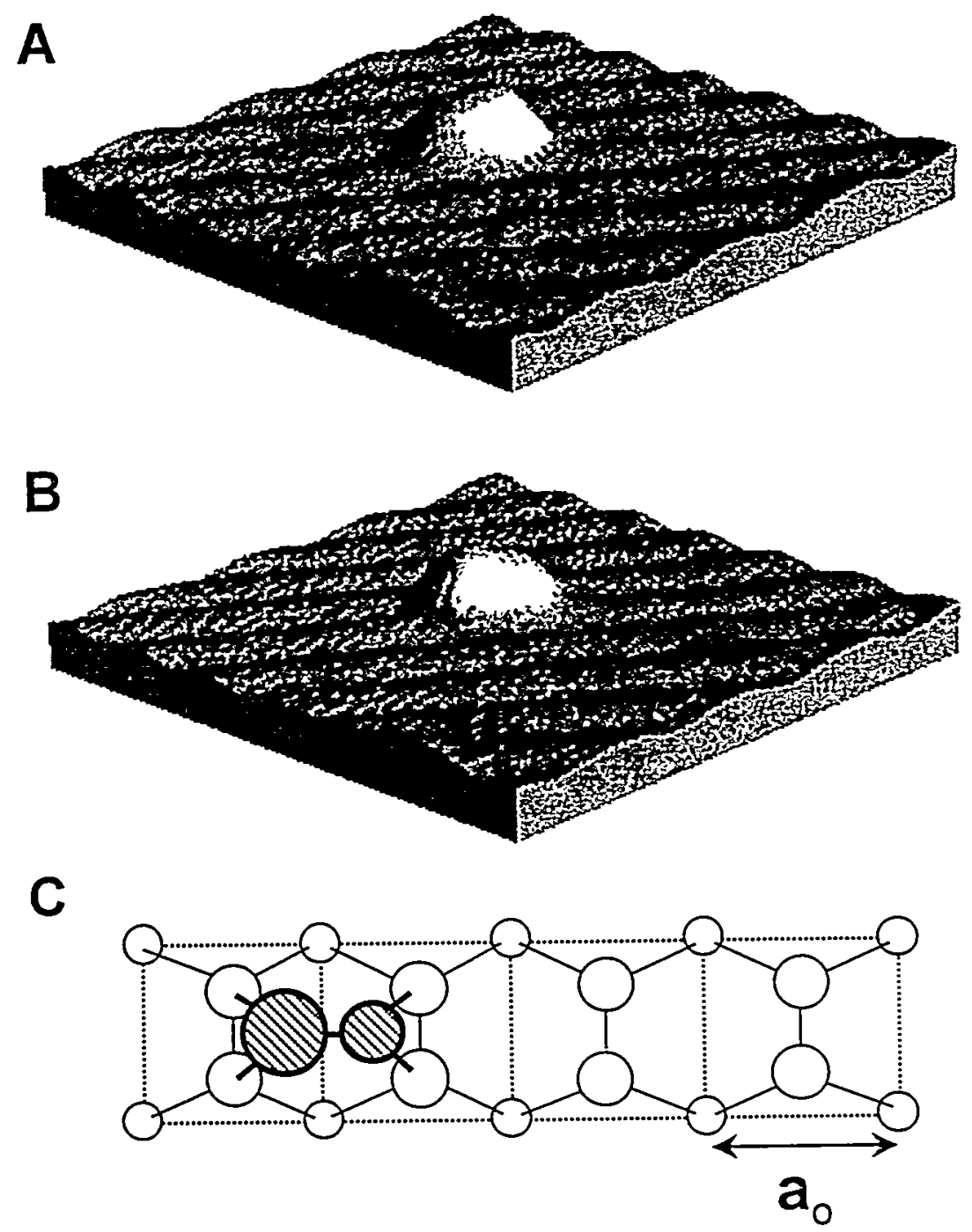

Fig. 1 Qin et al 

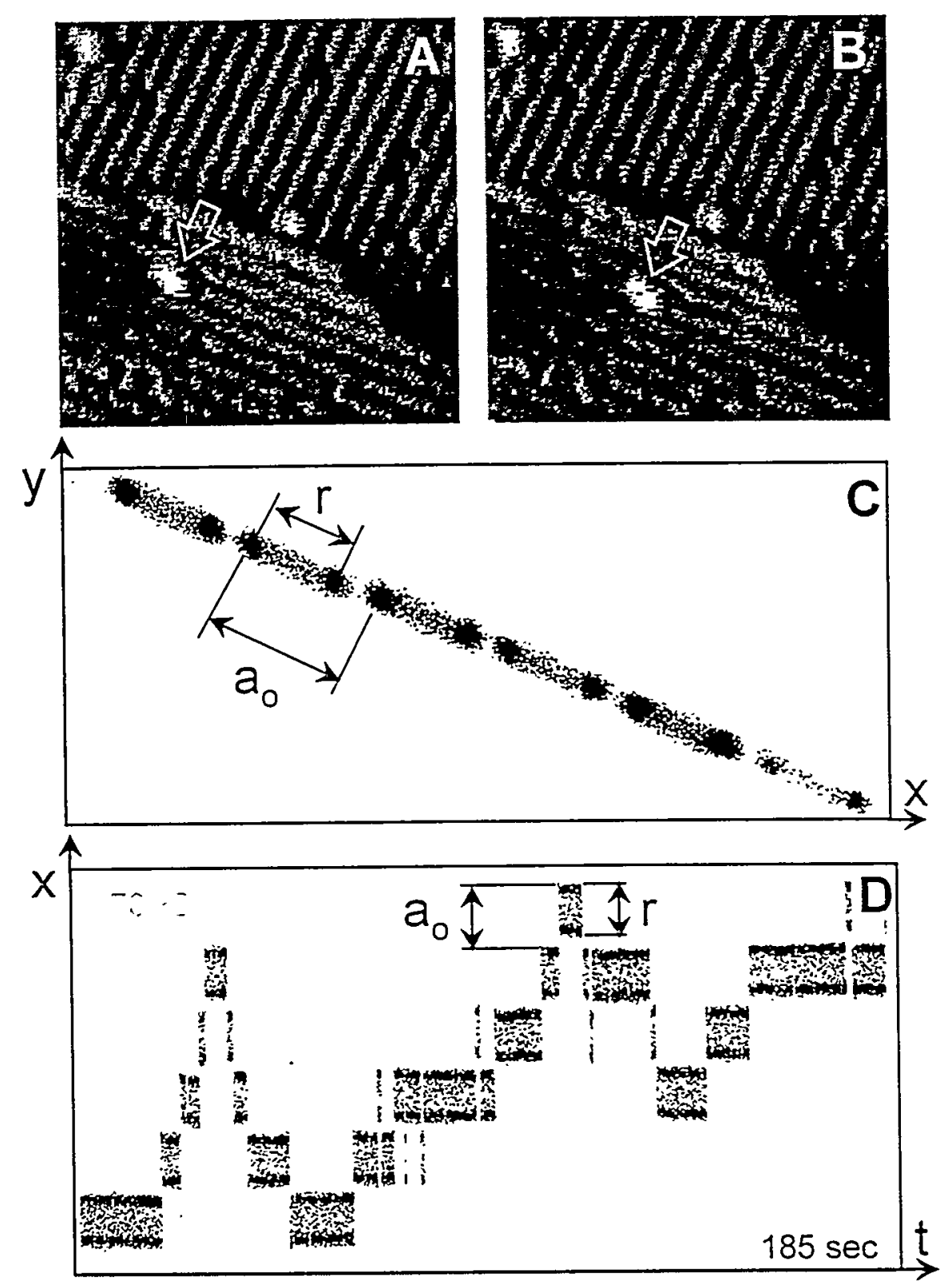

Fig. 2 Qin et al 


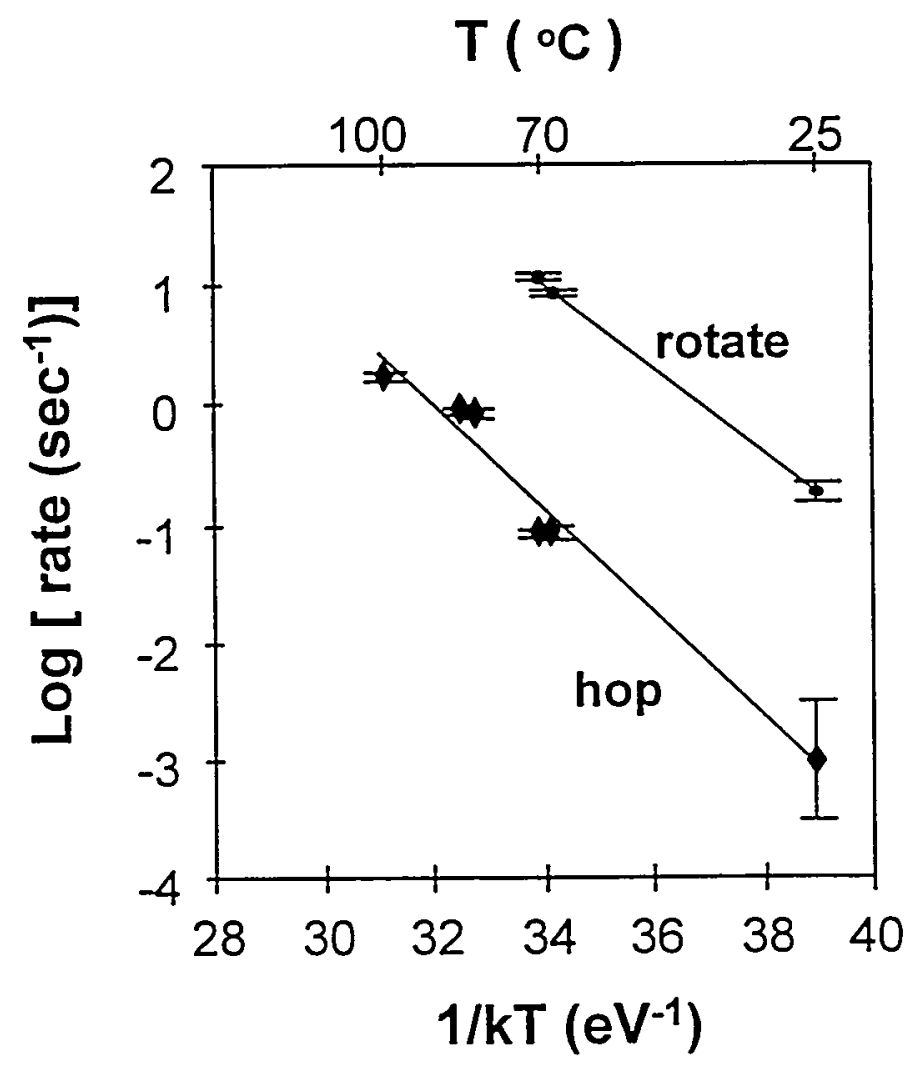

Fig. 3 Qin et al 

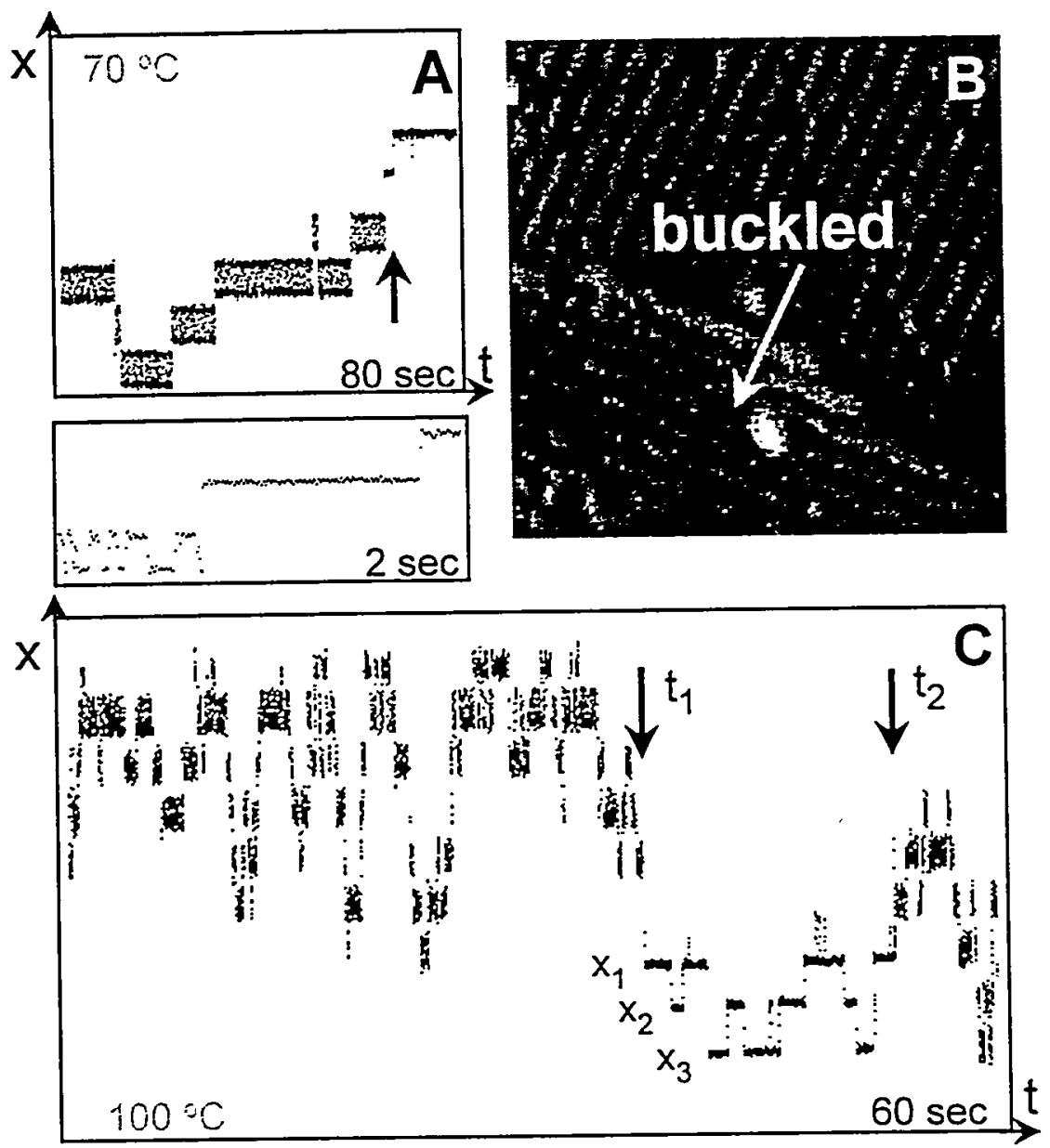

Fig. 4 Qin et al 